\title{
Hemorrhagic Cholecystitis in an Elderly Patient Taking Aspirin and Cilostazol
}

\author{
David S. Morris John R. Porterfield Mark D. Sawyer \\ Mayo Clinic, Rochester, Minn., USA
}

\section{Key Words}

Cholecystitis · Hemorrhagic cholecystitis · Phosphodiesterase inhibitor complications . Aspirin complications

\begin{abstract}
Hemorrhage is a rare complication of acute cholecystitis. Patients who develop this complication often are receiving anticoagulation therapy or have a pathologic coagulopathy. We present a case of an elderly patient who developed hemorrhagic cholecystitis while taking aspirin and cilostazol, a phosphodiesterase inhibitor. The patient underwent an emergent abdominal exploration. A large, blood-filled gallbladder was found along with a large hematoma between the liver and gallbladder. We also briefly review the literature regarding hemorrhagic cholecystitis, hemorrhage into the biliary tree, and hemorrhage as a complication of aspirin and phosphodiesterase inhibitor therapy.
\end{abstract}

\section{Case Report}

A 91-year-old woman presented to our emergency department with a 20-hour history of vague right-sided abdominal pain that was not associated with meals. She experienced nausea and vomiting without hematemesis. She had no change in her bowel movements and no melena.

The patient had a complex medical history, including a hospitalization for an exacerbation of congestive heart failure and pneumonia several months prior. She did not have a history of bleeding tendencies and no recent changes had been made to her daily medications. She had been taking cilostazol, a phosphodiesterase inhibitor, for the previous 4 years for lower extremity claudication and aspirin $(325 \mathrm{mg}$ ) for the previous 9 years for cardiovascular protective effects.

She was afebrile with a pulse of 86 beats per minute, blood pressure of $119 / 64 \mathrm{~mm} \mathrm{Hg}$, respirations of 18 breaths per minute, and oxygen saturation of $93 \%$ on room air. Her abdominal exam demonstrated right supraumbilical tenderness without involuntary guarding or rebound tenderness. Her leukocyte count was $18.1 \times 10^{9} / \mathrm{l}$, and her hemoglobin was $12.1 \mathrm{~g} / \mathrm{dl}$. Liver function tests, including AST, and total and direct bilirubin were normal, as were the patient's INR and aPTT. She was typed and crossed for 2 units of packed red blood cells but did not require transfusion of blood or platelets. 
The abdominal radiograph demonstrated no free intraabdominal air. A CT scan revealed inflammatory changes in the pericholecystic fat and gallbladder fossa, and IV contrast within the gallbladder lumen. IV contrast was also found in a small amount of free fluid around the liver. These findings were considered worrisome for hemorrhagic cholecystitis with perforation (fig. 1).

The patient was admitted to the surgical ICU for preoperative volume resuscitation. The aspirin and cilostazol were stopped. Given the patient's medical comorbidities, a cholecystostomy tube was initially considered. However, we recommended an urgent abdominal exploration because of the intraperitoneal hemorrhage.

The abdomen was explored via a right subcostal incision. Inspection of the right upper quadrant revealed approximately $350 \mathrm{ml}$ of blood. The gallbladder was intact yet necrotic and contained a large hematoma and countless 1- to 2-mm stones (fig. 2). Histology demonstrated acute hemorrhagic cholecystitis with cholelithiasis. After removal of the gallbladder, the abdomen was copiously irrigated with warm normal saline. No other abnormalities were found.

The patient recovered in the ICU for six days after the operation before being transferred to the floor. She experienced no postoperative bleeding episodes. She was discharged on postoperative day 21 after a hospital course which was dominated by the care of her medical comorbidities. Aspirin therapy was reinitiated uneventfully by her primary care physician at a lower dose $(81 \mathrm{mg})$ two months after this episode. The cilostazol was discontinued.

\section{Discussion}

Hemorrhage is a rare complication of acute cholecystitis. The mechanism is felt to be due to a transmural inflammatory process that causes erosion of the mucosa and ischemia. These processes then cause further mucosal breakdown, erosion into gallbladder vessels, and subsequent hemorrhage into the gallbladder lumen or the abdominal cavity [1]. We believe our patient experienced this same sequence of events, leading to her presentation.

Hemorrhage into the gallbladder lumen may occur for a variety of reasons, including obstructive cholecystitis, trauma, percutaneous interventions, biliary neoplasms, biliary parasites, or bleeding disorders. Our patient did not have a specific bleeding disorder; however, she was taking both aspirin and cilostazol, which create irreversible and reversible inhibition of platelet function, respectively. Several reports that link coagulopathy and hemorrhagic cholecystitis have been published [2-4]. Arteriosclerosis is thought to be a predisposing factor for gallbladder hemorrhage [5].

The patient with hemorrhage into the gallbladder may present in a variety of ways. The blood may clot inside the gallbladder, resulting in gallbladder distension and possibly perforation into the abdomen. The patient may then present with peritonitis. The blood may also clot inside the common bile duct, resulting in obstructive symptoms. Finally, the blood may enter into the bowel lumen, with subsequent hematemesis or melena. The patient in this case did have hemorrhage into the gallbladder and peritoneal cavity but did not present with frank peritonitis. This is likely due to the fact that there was no gross bilious contamination of the abdomen and the blood was contained to the right upper quadrant. The cystic duct was completely obstructed, preventing hemorrhage into the intestinal lumen. Thus our patient did not experience hematemesis or melena as often occurs with hemorrhage into the biliary tract.

The bleeding our patient experienced may have been exacerbated by cilostazol and aspirin therapy. Cilostazol is a phosphodiesterase inhibitor which decreases platelet aggregation. It is used in a variety of settings, including lower extremity claudication, after coronary stent placement, or cerebrovascular accident prevention. Cilostazol may offer chronotropic benefit in the treatment of patients with bradyarrhythmia [6]. Results of 
investigation into the effect of cilostazol on hemostasis have been mixed, with at least one study demonstrating prolonged bleeding [7], while another failed to show a similar effect [8]. Platelet counts have not been found to be adversely affected [9]. Aspirin, on the other hand, has been shown clearly to prolong bleeding time and affect platelet aggregation $[8$, $10,11]$.

The treatment of hemorrhagic cholecystitis is cholecystectomy, or alternatively cholecystostomy in selected patients. As in our patient's case, with intensive perioperative care, even patients of advanced age with multiple significant medical comorbidities can be successfully treated surgically. We are certainly thankful our 91-year-old patient ultimately recovered and was discharged after extensive efforts on the part of our medical and surgical services, but realize that this may not always be the case. Cholecystostomy should be reserved for those patients too unstable for the operating room and in whom adequate drainage can be accomplished.

\section{Conclusion}

Our patient experienced hemorrhagic cholecystitis as a rare complication of acute cholecystitis. While impossible to prove definitively, it is reasonable to suggest that treatment with aspirin and cilostazol contributed to the degree of hemorrhage. To our knowledge, this is the first report of hemorrhagic cholecystitis associated with these drugs.

Fig. 1. Arterial phase contrast CT scan of the abdomen. The asterisk marks the mixed-density hemorrhagic fluid within the gallbladder (65 Hounsfield units).

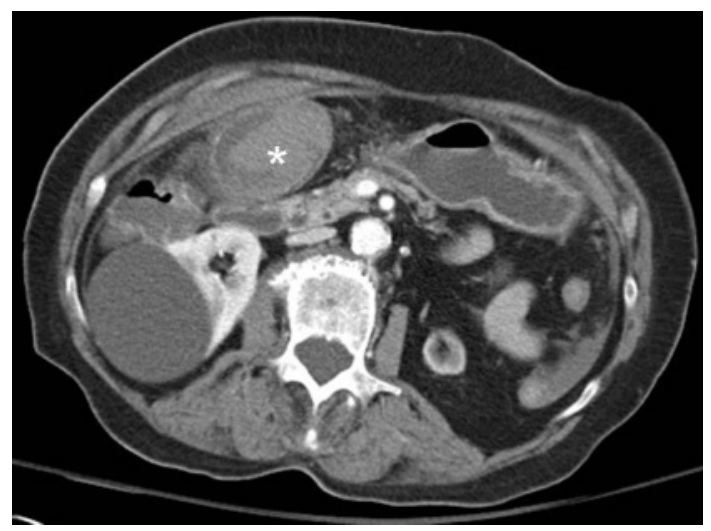


Fig. 2. Intraoperative photo demonstrating a tense, distended gallbladder and focal hemorrhage of the gallbladder wall.

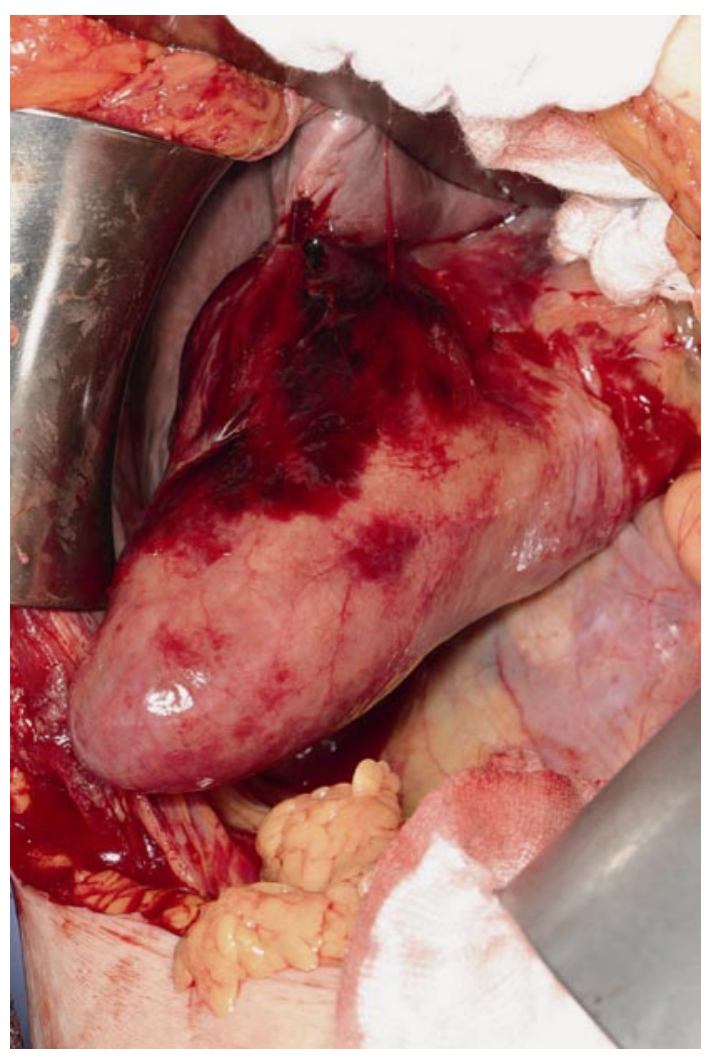




\section{References}

1 Gremmels JM, Kruskal JB, Parangi S, Kane RA: Hemorrhagic cholecystitis simulating gallbladder carcinoma. J Ultrasound Med 2004;23:993-995.

2 Brawner J, Trivedi H, Sataline LR: Hemocholecyst. Report of a case associated with anticoagulation therapy. Ohio State Med J 1966;62:1028-1030.

-3 Avakoff JC, Wolfman EF Jr: Hemocholecyst: report of two cases, one associated with steroid administration. Surgery 1969;66:830-834.

4 Butterfield WC, Choi U: Hemobilia associated with hemorrhagic cholecystitis in a patient with alcoholic cirrhosis. Conn Med 1971;35:295 passim.

5 Hudson PB, Johnson PP: Hemorrhage from the gallbladder. N Engl J Med 1946;234:438-441.

6 Atarashi H, Endoh Y, Saitoh H, Kishida H, Hayakawa H: Chronotropic effects of cilostazol, a new antithrombotic agent, in patients with bradyarrhythmias. J Cardiovasc Pharmacol 1998;31:534-539.

7 Uehara S, Hirayama A: Effects of cilostazol on platelet function. Arzneimittelforschung 1989;39:1531-1534.

8 Tamai Y, Takami H, Nakahata R, Ono F, Munakata A: Comparison of the effects of acetylsalicylic acid, ticlopidine and cilostazol on primary hemostasis using a quantitative bleeding time test apparatus. Haemostasis 1999;29:269-276.

-9 Ikeda Y, Kikuchi M, Murakami H, et al: Comparison of the inhibitory effects of cilostazol, acetylsalicylic acid and ticlopidine on platelet functions ex vivo. Randomized, double-blind cross-over study. Arzneimittelforschung 1987;37:563566.

10 Fiore LD, Brophy MT, Lopez A, Janson P, Deykin D: The bleeding time response to aspirin. Identifying the hyperresponder. Am J Clin Pathol 1990;94:292-296.

-11 Sonksen JR, Kong KL, Holder R: Magnitude and time course of impaired primary haemostasis after stopping chronic low and medium dose aspirin in healthy volunteers. Br J Anaesth 1999;82:360-365. 